Research Article

\title{
Performance Degradation Assessment of Rotary Machinery Based on a Multiscale Tsallis Permutation Entropy Method
}

\author{
Yong Chen $(\mathbb{D}$, Mian Jiang $(\mathbb{D}$, and Kuanfang He $\mathbb{B}$ \\ Foshan University, School of Mechatronics Engineering and Automation, Foshan, China \\ Correspondence should be addressed to Mian Jiang; mjiang@fosu.edu.cn
}

Received 16 February 2021; Revised 9 March 2021; Accepted 17 March 2021; Published 26 March 2021

Academic Editor: Yi Qin

Copyright (c) 2021 Yong Chen et al. This is an open access article distributed under the Creative Commons Attribution License, which permits unrestricted use, distribution, and reproduction in any medium, provided the original work is properly cited.

\begin{abstract}
Methods based on vibration analysis are currently regarded as the most conclusive means for fault diagnosis and health prognostics in rotary machinery. However, changing working conditions mean that the vibration signals originating from rotary machinery exhibit different levels of complexity. This complexity leads to increased difficulty in constructing health indicators (HIs). In this paper, we propose a multiscale Tsallis permutation entropy (MTPE) to construct the HIs of rotary machinery under different working conditions. MTPE values are a function of an entropy index and scale, which have the universality for handling the complexity of a permutated time series. The health condition of the rotary machinery was effectively represented by the MTPEs in conditional monitoring; the initial point of the unhealthy stage was found using the $3 \sigma$ interval. This was set as the alarm threshold according to the varying HI trend. Once this was established, dividing the stages into two-stage health stages (HS) was straightforward. Using a rolling bearing, a run-to-failure experiment was conducted and results suggested that the proposed method effectively assessed the status of the rotary machinery. Taken together, this study provided a novel complexity measure based on a methodology for constructing the HIs of rotary machinery and enriches conditional monitoring theory.
\end{abstract}

\section{Introduction}

Condition-based maintenance (CBM) is effective in reducing unnecessary maintenance operations and improving the reliability of rotary machinery [1]. One of the major tasks in $\mathrm{CBM}$ is health prognostics, which aims to predict the remaining useful life (RUL) of rotary machinery based on its historical and ongoing degradation trends. Extensive research has been done into data-driven methods for CBM; from this work, vibration analysis has emerged as the most conclusive method for fault diagnosis and health prognostics in rotary machinery [2-6]. The prognostic program generally consists of four technical processes: data acquisition, health indicator (HI) construction, health stage (HS) division, and RUL prediction [1]. As a representative of the health condition of the machinery, HI performance has a great influence on the effect of prognostics. In recent years, synthesized HIs have received much attention, owing to their impressive results for RUL prediction $[7,8]$. These are usually constructed by a combination of signal processing techniques and artificial intelligent (AI) techniques [9]. Along with the measured vibration signals, both time- [10] and frequency-domain features [11] are widely used to analyze the status of the rotary machinery.

Conventional indicators, such as kurtosis [12] and skewness, fluctuate drastically and affect the assessment of the machinery running status [9]. Root mean square (RMS) [13-16] values are stable during the healthy stage and will usually experience an "increase-decrease-increase" trend during the unhealthy stage. When only using the RMS values, the fault threshold is difficult to determine. With the development of the FFT algorithm, frequency-domain features are also utilized as criteria to identify the health condition of the rotary machinery. By identifying the aroused fault frequency by the fault across a wide frequency band, the defect is better visualized. However, in some cases (e.g., inner ring defect of bearing and complex defect), the complex spectrum makes the frequency-domain features less effective [9]. Moreover, an additional disadvantage is that the construction of frequencydomain features requires significant expertise. 
AI techniques have also been used to establish HIs and assess the overall health conditions. These indicators are generally constructed by fusing multiple features, presenting a virtual description of the degradation trends of the machinery itself. For instance, Chen [17] constructed fused feature vectors using multiple, two-layer sparse autoencoder neural networks for bearing fault classification. Guo et al. [8] constructed a health index for bearing prognostics by fusing multiple features using a recurrent neural network. Using the input of an autoregression model to predict bearing states, Qian [18] extracted features by implementing a recurrence quantification analysis. The vibration features processed by the isometric feature mapping technique (ISOMAP) were proposed by Benkedjouh [19] to be the inputs of a support vector regression (SVR) model to predict the bearing RUL. Ocak et al. [20] fused multiple features using the hidden Markov model (HMM) in the healthy stage. Keshtegar et al. [21] developed the multi-extremummodified response basis model for dynamic, nonlinear responses of failure capacities for a turbine blisk. Fei et al. [22] introduced an enhanced network learning method to improve the modeling precision and simulation efficiency in flexible mechanism reliability evaluation. Han et al. [23] proposed a probability-based prediction method to predict the service safety life of aero engine turbine blades. As indicated here, AI techniques have been widely used; however, past approaches have revealed that their shallow architectures restrict their capability of learning complicate nonlinear relationships [24, 25]. Moreover, given the highly nonlinear characteristics in the degradation process of rolling bearings [26], more powerful methodologies for the construction of HIs are needed.

During the degradation process of rotary machinery, increasingly complicated behaviors have been shown in the measured vibration signals used for health prognosis. The advent of better computational efficiency has allowed the complexity measure to calculate the complexity values in the vibration signals. This then allows for the assessment of the working status of the rotary machines. The complexity of a signal time series can be described by the Lempel-Ziv Complexity (LZC) [27, 28], Approximation Entropy (ApEn) [29], permutation entropy (PE) [30-32], and multifeature entropy $[33,34]$. These LZC values describe the degree of regularity of the vibration signals, and the quantitative relationship between the LZC values and the defect size, and the severity level. Moreover, this approach has already been established in rolling bearings. Despite this, using the definite calculation of the vibration signals based on the LZC index will lead in incorrect judgements for health conditions of rotary machinery in practical engineering. This is because the fault feature information is often submerged in noise [28]. For instance, Yan et al. [29] presented the ApEn to give the measurement of the regularity for mechanical structure signals. However, the ApEn greatly relies on data length; as a result, it will produce inaccurate estimation by using the ApEn to deal with a short time series. Compared to either LZC or ApEn, PE (multiscale PE) is a novel method used to quantify the degree of complexity and was originally proposed by Bandt and Pompe [30]. Fei et al. [33] introduced the multifeature entropy distance method for the process character analysis and subsequent diagnosis of rolling bearing faults. This was achieved by integrating four information entropies and two signal types. Moreover, the WCFSE-FSVM method [34] has also been proposed; it consists of fusing the advantages of the wavelet correlation feature scale entropy with those of the fuzzy support vector machine. However, both the PE and multiscale PE [35] have undeniable shortcoming [36]. More specifically, it has difficulty in handling the complexity of a permutated time series for rotary machinery under different working conditions.

In this paper, we proposed a MTPE analysis for conditional monitoring in the degradation process of rotary machinery. The MTPE values are a function of both the entropy index and scale, which have the universality for dealing with the complexity of a permutated time series. MTPE analysis has the capacity to construct HIs of the rotary machinery under different working conditions. The MTPE was used to construct these HIs; the effects of entropy index, scale, embedding dimension, and time delay for the time series from the standard logistic map [37] were also assessed. Using the $3 \sigma$ interval as the alarm threshold, the initial point of the unhealthy stage was determined; the process was then divided into two health stages according to the varying trends of the HIs. For the unhealthy stage, the HI trend regarding the degradation process was established using a double exponential function together with an ARMAX model. A run-to-failure experiment for the rolling bearing was then designed to evaluate the capability of the proposed method. Results suggested that that the proposed method was effective for the assessment of the rotary machinery.

\section{Performance Degradation Assessment Based on the MTPE Method}

2.1. Multiscale Tsallis Permutation Entropy. Tsallis permutation entropy (TPE) is a generalization of the Boltzmann-Gibbs-Shannon entropy and has been proved to be efficient and suitable for multifractal systems [36]. The main difference between TPE and PE based on Shannon entropy is that the entropic index embedded in TPE is used to measure the nature of complex time series under different conditions [36]. The TPE is a special form of PE and derives from ordinal patterns. Given the original time series $\mathbf{x}_{1}, \mathbf{x}_{2}, \ldots, \mathbf{x}_{\mathbf{r}}$, the subtime series $\mathbf{y}_{1}^{\mathbf{s}}, \mathbf{y}_{2}^{\mathbf{s}}, \ldots$ is constructed through the scale s. Each subtime series

$$
\mathbf{y}_{\mathbf{j}}^{\mathbf{s}}=\frac{1}{\mathbf{s}} \sum_{\mathbf{i}=(\mathbf{j}-1) \mathbf{s}+1}^{\mathbf{j s}} \mathbf{x}_{\mathbf{i}}, \quad \mathbf{j}=1,2, \ldots, \mathbf{N},
$$

where $\mathbf{N}=[\mathbf{r} / \mathbf{s}]$ denotes the round-off number of $\mathbf{r} / \mathbf{s}$ and $\mathbf{s}$ is the scale. For $\mathbf{s}=1$, the time series $\mathbf{y}^{1}$ is the original time series $\mathbf{x}_{1}, \mathbf{x}_{2}, \ldots, \mathbf{x}_{r}$. The length of each subtime series is $\mathbf{N}=[\mathbf{r} / \mathbf{s}]$.

For any segment $\mathbf{y}^{\mathbf{s}}$, the value of TPE is computed according to previously published work [31]. According to the Takens-Maine theorem, the phase space of time series $\mathbf{y}^{\mathbf{s}}(\mathbf{i}), \mathbf{i}=1,2, \ldots, \mathbf{N}$ is reconstructed as 


$$
\left\{\begin{array}{l}
\mathbf{Y}^{\mathbf{s}}(1)=\left\{\mathbf{y}^{\mathbf{s}}(1), \mathbf{y}^{\mathbf{s}}(1+\tau), \ldots, \mathbf{y}^{\mathbf{s}}(1+(\mathbf{m}-1) \tau)\right\}, \\
\vdots \\
\mathbf{Y}^{\mathbf{s}}(\mathbf{i})=\left\{\mathbf{y}^{\mathbf{s}}(\mathbf{i}), \mathbf{y}^{\mathbf{s}}(\mathbf{i}+\tau), \ldots, \mathbf{y}^{\mathbf{s}}(\mathbf{i}+(\mathbf{m}-1) \tau)\right\}, \\
\vdots \\
\mathbf{Y}^{\mathbf{s}}(\mathbf{N}-(\mathbf{m}-1) \tau)=\left\{\mathbf{y}^{\mathbf{s}}(\mathbf{N}-(\mathbf{m}-1) \tau), \mathbf{y}^{\mathbf{s}}(\mathbf{N}-(\mathbf{m}-2) \tau), \ldots, \mathbf{y}^{\mathbf{s}}(\mathbf{N})\right\},
\end{array}\right.
$$

where $\mathbf{m}$ is the embedded dimension and $\tau$ is the time delay. Then, the $\mathbf{m}$ number of real values contained in each $\mathbf{y}^{\mathbf{s}}(\mathbf{i}), \mathbf{i}=1,2, \ldots, \mathbf{N}$ are arranged in increasing order as

$$
\mathbf{y}^{\mathbf{s}}\left(\mathbf{i}+\left(\mathbf{k}_{1}-1\right) \tau\right) \leq \mathbf{y}^{\mathbf{s}}\left(\mathbf{i}+\left(\mathbf{k}_{2}-1\right) \tau\right) \leq \cdots \leq \mathbf{y}^{\mathbf{s}}\left(\mathbf{i}+\left(\mathbf{k}_{\mathbf{m}}-1\right) \tau\right) .
$$

If two or more elements in each $\mathbf{y}^{\mathbf{s}}(\mathbf{i})$ have the same value, for example, $\mathbf{y}^{\mathbf{s}}\left(\mathbf{i}+\left(\mathbf{k}_{\mathbf{m}-1}-1\right) \tau\right)=\mathbf{x}_{\mathbf{i j}}\left(\mathbf{i}+\left(\mathbf{k}_{\mathbf{m}}-1\right) \tau\right)$, their original position is sorted for $\mathbf{k}_{\mathbf{m}-1} \leq \mathbf{k}_{\mathbf{m}}$, and $\mathbf{y}^{\mathbf{s}}(\mathbf{i}+$ $\left.\left(\mathbf{k}_{\mathbf{m}-1}-1\right) \tau\right) \leq \mathbf{y}^{\mathbf{s}}\left(\mathbf{i}+\left(\mathbf{k}_{\mathbf{m}}-1\right) \tau\right)$ is recorded. Then, the vector $\mathbf{y}^{\mathbf{s}}(\mathbf{i})$ is mapped onto a group of symbols as

$$
\mathbf{K}(\mathbf{q})=\left(\mathbf{k}_{1}, \mathbf{k}_{2}, \ldots, \mathbf{k}_{\mathbf{m}}\right),
$$

where $\mathbf{l}=1,2, \ldots, \mathbf{K}$ and $\mathbf{K} \leq \mathbf{m} !$. $\mathbf{S}(\mathbf{l}), \mathbf{l}=1,2, \ldots, \mathbf{K}$, is one of the $\mathbf{m}$ ! symbol permutations, if $\mathbf{P}_{1}^{\mathbf{s}}, \mathbf{P}_{2}^{\mathbf{s}}, \ldots, \mathbf{P}_{\mathbf{K}}^{\mathbf{s}}$ are used to denote the probability distribution of each symbol sequence, respectively. The PE of $\mathbf{y}^{\mathrm{s}}$ is defined based on the $\mathbf{K}$ symbol permutations as

$$
\operatorname{MTPE}(\mathbf{q}, \mathbf{s})=\frac{1-\sum_{\mathbf{i}=1}^{\mathbf{K}}\left(\mathbf{P}_{\mathbf{i}}^{\mathbf{s}}\right)^{\mathbf{q}}}{\mathbf{q}-1} .
$$

Then, the multiscale permutation entropy (MPE) PE (s) for the original time series is given based on the Shannon entropy. However, the TPE is not simply the generalization of the Shannon permutation entropy. Given the entropy index $\mathbf{q}$, the MTPE is defined as follows:

$$
\operatorname{MTPE}(\mathbf{q}, \mathbf{s})=\frac{1-\sum_{\mathbf{i}=1}^{\mathrm{K}}\left(\mathbf{P}_{\mathbf{i}}^{\mathbf{s}}\right)^{\mathbf{q}}}{\mathbf{q}-1},
$$

where $\mathbf{P}_{i}^{\mathbf{s}}$ is calculated based on the time series $\mathbf{y}^{\mathbf{s}}$. Obviously, the value of MTPE in equation (6) is the function of the entropy index $\mathbf{q}$ and the scale $\mathbf{s}$. The purpose of MTPE is to study the universality for dealing with the complexity of the permutated time series. Since its values are the function of the entropy index and scale, MTPE analysis has the capacity to construct the HIs of the rotary machinery under different working conditions.

2.2. Parameter Effects on MTPEs. To study the effects of different parameters on MTPE values, the logistic map shown in [37] was used as an exemplary object. The logistic map is often used to describe the change of quantity with increasing time, which is written as the simple mathematical model as follows:

$$
\mathbf{x}_{\mathbf{n}+1}=\mu \mathbf{x}_{\mathbf{n}}\left(1-\mathbf{x}_{\mathbf{n}}\right) \text {, }
$$

where $\mathbf{n}=1,2, \ldots, \mathbf{x}_{\mathbf{n}} \in[0,1]$ and $\mu$ is the control parameter. The bifurcate evolutional process of the logistic map with $2.5<\mu<4$ and chaotic behavior with $3.5699<\mu<4$ are shown in $[31,37]$, respectively. In this paper, the time series using equation (7) is generated with 4096 points by starting from $\mathbf{x}_{0}=0.65$, where chaotic behavior occurred with $\mu=3.75$. The effects of entropy index $q$, embedding dimension $m$, and time delay $\tau$ on the MTPEs are discussed with increasing scale in Figures 1-5. First, the MPE and MTPE $(q=0.98)$ were compared in Figure 1 . Given these results, it was concluded that MTPE was more sensitive than MPE with changing scales.

The effects of the positive entropy index $q$ on MTPEs are shown in Figure 2; the effects of the negative entropy index are shown in Figure 3. All MPTEs and MPEs first increased, after which they slowly decreased with increasing scales. It is obvious that the values of the entropy index had a great impact on MTPE amplitude. All MTPEs had maximum amplitudes when using a scale of two; amplitudes then decreased with increasing scales larger than two. Other parameters that affected the MTPE calculations were the embedding dimension $m$ and the time delay $\tau$. The relationship between the MTPE values and embedding dimension is illustrated in Figure 4, where the embedding dimensions were selected to be $m=3-8$. Similarly, the relationship between the MTPE values and time delay is illustrated in Figure 5, where the time delays were selected to be $\tau=1-4$. According to the analysis presented in previous work [31], we selected $m=6$ and $\tau=3$ to compute the MTPEs of the vibration signals in the degradation process of the rolling bearing.

2.3. Process of Assessing Performance Degradation. The bearing performance degradation assessment based on our MTPE analysis included several steps: (1) acquiring vibration signals, (2) calculating MTPEs, and (3) calculating HIs by normalization. The procedures for assessing the degradation in bearing performance are shown in Figure 6.

2.3.1. Vibration Signals Acquisition. During the degradation process of the rotary machinery, the time series of the vibration signals were collected at time points $\mathbf{t}_{1}, \mathbf{t}_{2}, \ldots, \mathbf{t}_{\mathbf{n}}$, where $\mathbf{n}$ denotes the maximum number of vibration signals sampled in the process. Then, a set of vibration signals $\mathbf{X}_{1}, \mathbf{X}_{2}, \ldots, \mathbf{X}_{\mathbf{n}}$ is collected to reflect the dynamic changes of the rotary machinery during the degradation process. The length of each $\mathbf{X}_{\mathbf{i}}$ is assumed to be $\mathbf{r}$ and $\mathbf{X}_{\mathbf{i}}=\left\{\mathbf{X}_{\mathbf{i} 1}, \mathbf{X}_{\mathbf{i} 2}, \ldots\right.$, $\left.\mathbf{x}_{\text {ir }}\right\}, \mathbf{i}=1,2, \ldots, \mathbf{n}$.

2.3.2. Calculation of MTPEs. The nonlinear dynamics of the rotary machinery are discriminated by computing the MTPEs of the time series of measured vibration signals. 


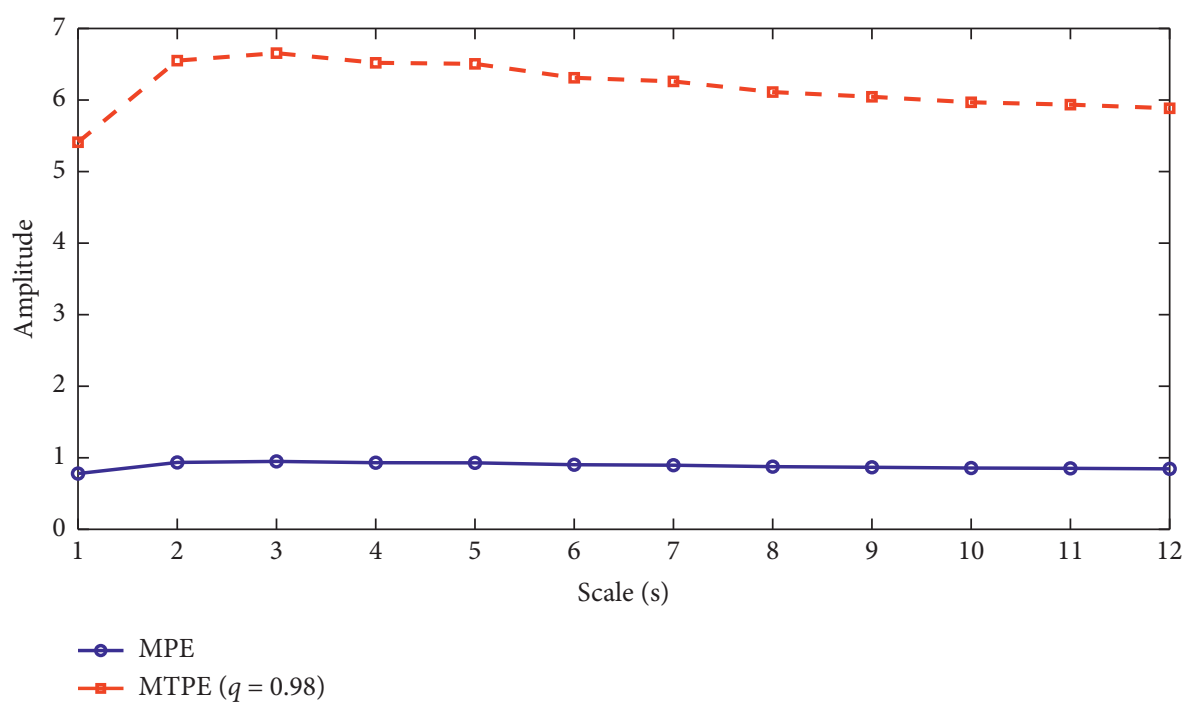

Figure 1: Comparison of MPE and MTPE with different scales.

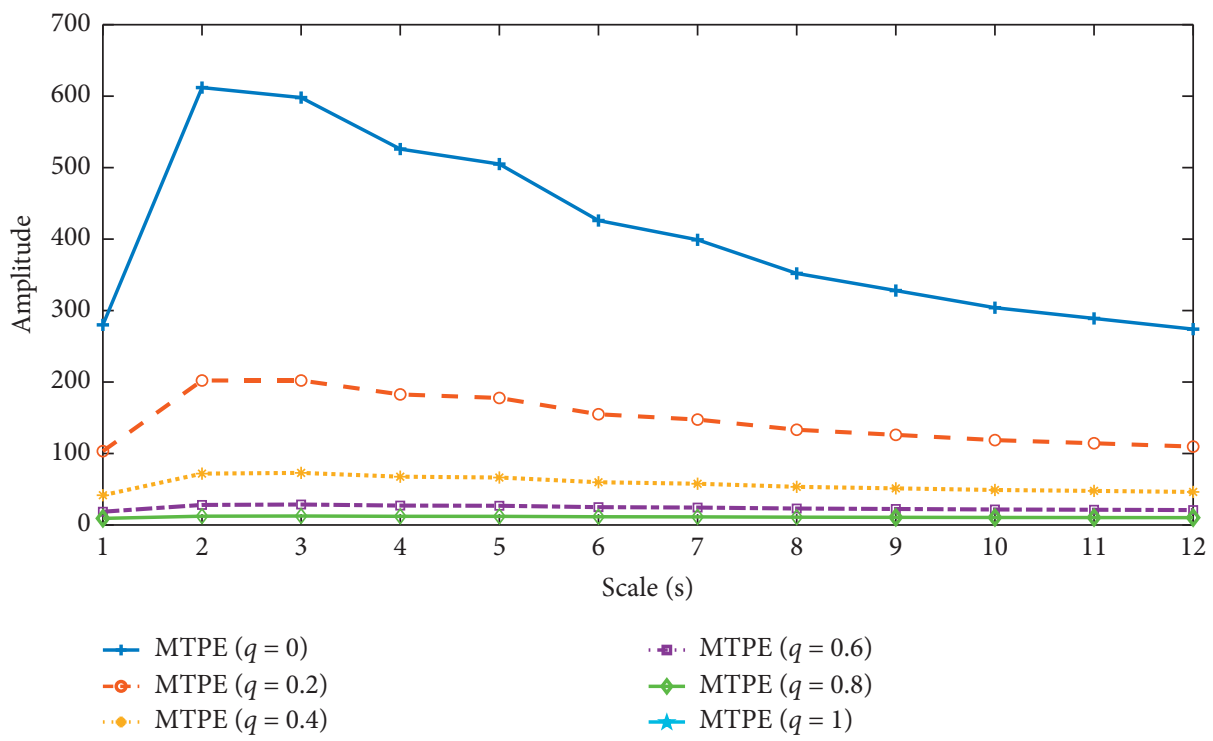

FIgUre 2: Effects of positive entropy index $q$ on MTPEs.

Assuming that the time series $\mathbf{X}_{\mathbf{i}}$ at the time $\mathbf{t}_{\mathbf{i}}$ is $\mathbf{x}_{\mathbf{i} 1}, \mathbf{x}_{\mathbf{i} 2}, \ldots, \mathbf{x}_{\mathbf{i r}}$, the value of MTPE with entropy index $\mathbf{q}$ and scale $\mathbf{s}$ is calculated as $\operatorname{MTPE}_{\mathbf{i}}(\mathbf{q}, \mathbf{s})$ according to equation (6). Then, the MTPE values for $\mathbf{X}_{1}, \mathbf{X}_{2}, \ldots, \mathbf{X}_{\mathbf{n}}$ are given as the set $\left\{\operatorname{MTPE}_{1}(\mathbf{q}, \mathbf{s}), \operatorname{MTPE}_{2}(\mathbf{q}, \mathbf{s}), \ldots, \operatorname{MTPE}_{\mathbf{n}}(\mathbf{q}, \mathbf{s})\right\}$.

2.3.3. Calculation of HIs by Normalization. The HIs $\delta_{1}, \delta_{2}, \ldots, \delta_{\mathbf{n}}$ are obtained by the normalized process for the set of MTPEs:

$$
\delta_{\mathbf{i}}(\mathbf{q}, \mathbf{s})=\frac{\operatorname{MTPE}_{\mathbf{i}}(\mathbf{q}, \mathbf{s})-\min \operatorname{MTPE}(\mathbf{q}, \mathbf{s})}{\max \operatorname{MTPE}(\mathbf{q}, \mathbf{s})-\min \operatorname{MTPE}(\mathbf{q}, \mathbf{s})},
$$

$$
\mathbf{i}=1,2, \ldots, \mathbf{n},
$$

where $\delta_{\mathbf{i}}(\mathbf{q}, \mathbf{s})$ denotes the $i$ th $\mathrm{HI}, \min \operatorname{MTPE}(\mathbf{q}, \mathbf{s})=$ $\min \left\{\operatorname{MTPE}_{1}(\mathbf{q}, \mathbf{s}), \ldots, \operatorname{MTPE}_{\mathbf{n}}(\mathbf{q}, \mathbf{s})\right\}, \max \operatorname{MTPE}(\mathbf{q}, \mathbf{s})=$ $\max \left\{\operatorname{MTPE}_{1}(\mathbf{q}, \mathbf{s}), \ldots, \operatorname{MTPE}_{\mathbf{n}}(\mathbf{q}, \mathbf{s})\right\} . \quad$ The HIs $\delta_{1}, \delta_{2}, \ldots, \delta_{\mathrm{n}}$ are used to represent the health condition during the degradation process of rolling bearing.

2.3.4. Healthy Stage Division. According to the varying degradation trend of HIs, the whole lifetime of the rolling bearing was divided into two or more different health stages. The HIs in the unhealthy stage were fitted using the analysis of degradation trends. A strategy for the two-stage division was used by identifying whether the HIs exceeded a constant alarm threshold.

\section{Experimental Verification}

3.1. Experimental System. A run-to-failure experiment for the rolling bearing was next designed and conducted to 


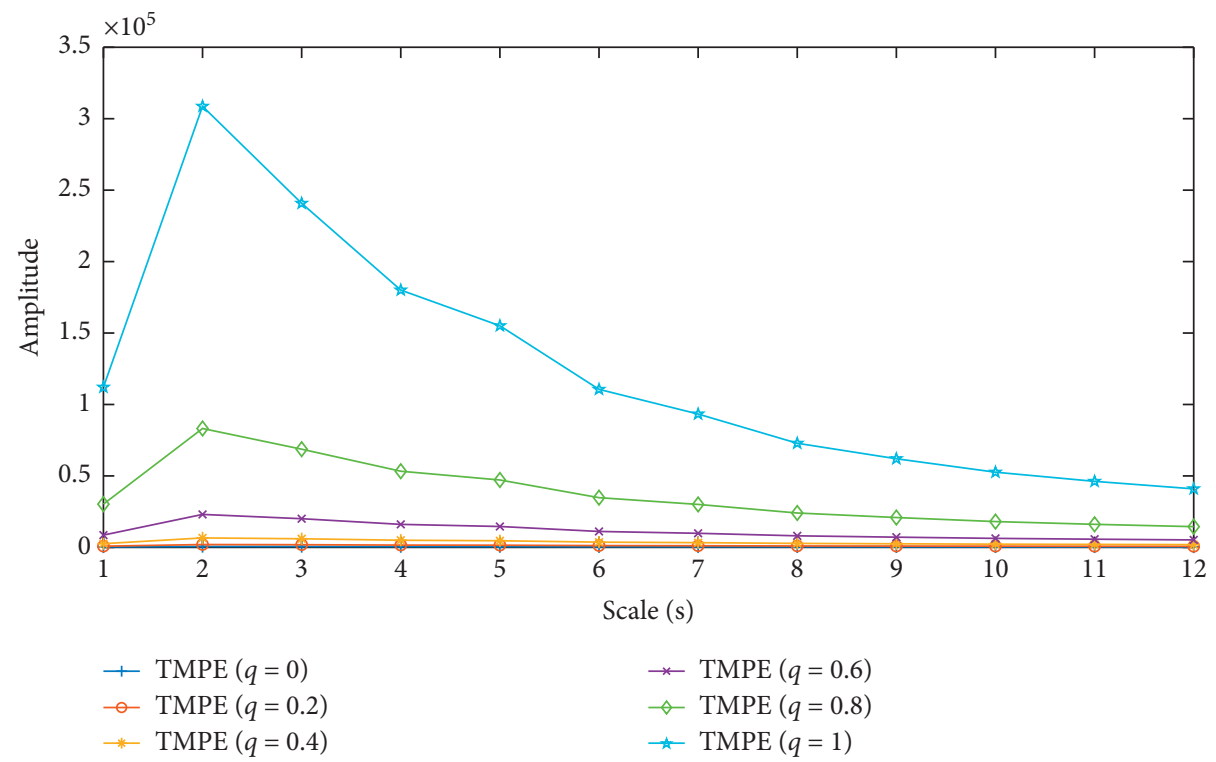

FIgUre 3: Effects of negative entropy index $q$ on MTPEs.

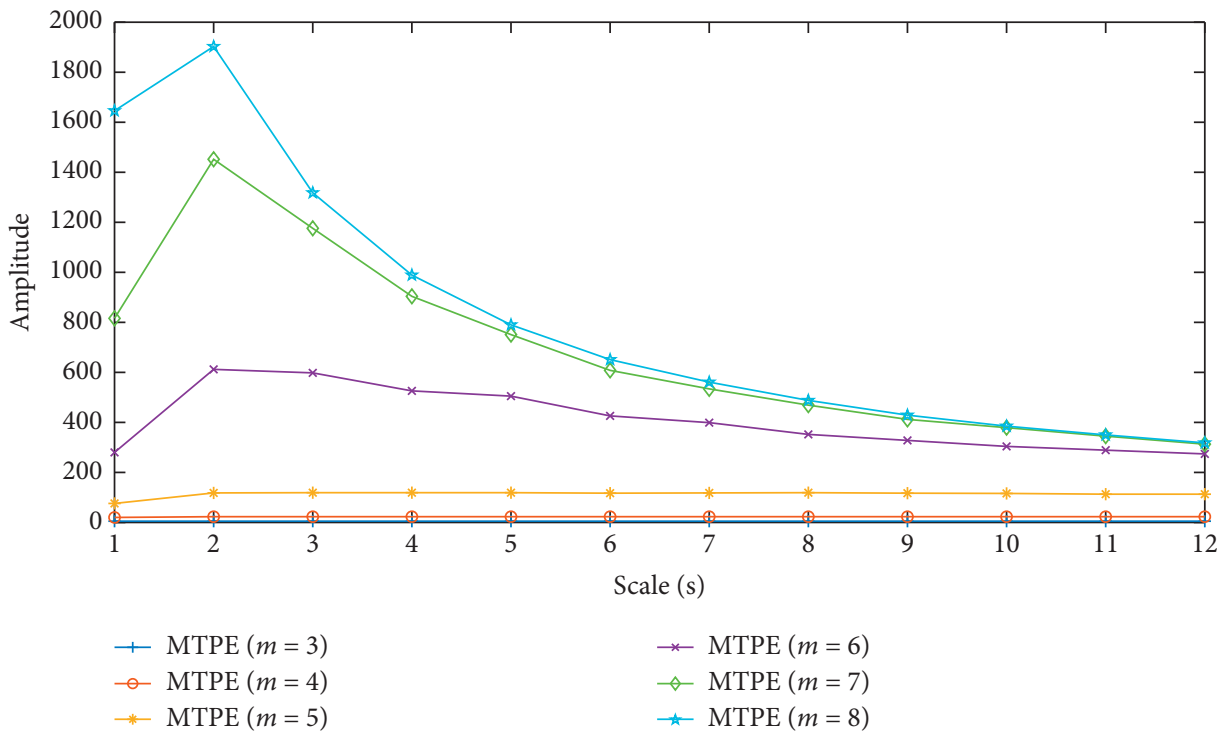

Figure 4: Effects of embedding dimension $m$ on MTPEs.

investigate the proposed method. The ABLT-7 bearing test system is shown in Figure 7, which consisted of a supporting pillow block, a test bearing with supporting housing, control electronics, and other features. The input-shaft of the test system is connected with an electromotor through a V-type belt, where the speed of the motor was adjusted using the integrated speed controller.

An accelerometer (B\&K4507B) and an acoustic emission sensor (B\&K4190L) were installed on the testing bearing housing to measure the vibrations. The resulting data were acquired through a multichannel analyzer (B\&K3560C) and Pulse software. A virtual instrumentation system was established by VC to obtain the timedomain feature of the vibrations, thereby ensuring the safety of the testing system.
In the run to failure experiment, a UCP207 insert bearing was tested. The radial load went from $5040 \mathrm{~N}$ for the first two hours to $8020 \mathrm{~N}$ for the remainder of its life; rotational speed was $3000 \mathrm{rpm}$. The sample frequency was $20 \mathrm{kHz}$ and the length of each vibration time series for sampling was set to 4096 . Vibration signals were obtained during the experiment at an interval of every $5 \mathrm{~min}$. In total, 530 data segments were collected to conduct the MTPE analysis and determine the status of the rolling bearing.

3.2. Vibration Signals Denoising and Analysis. As a widely used data denoising technique, we used wavelet transform to denoise the set of vibration signals $\mathbf{X}_{1}, \mathbf{X}_{2}, \ldots, \mathbf{X}_{\mathbf{n}}$ [27]. The transform was a linear transform that analyzed nonstationary 


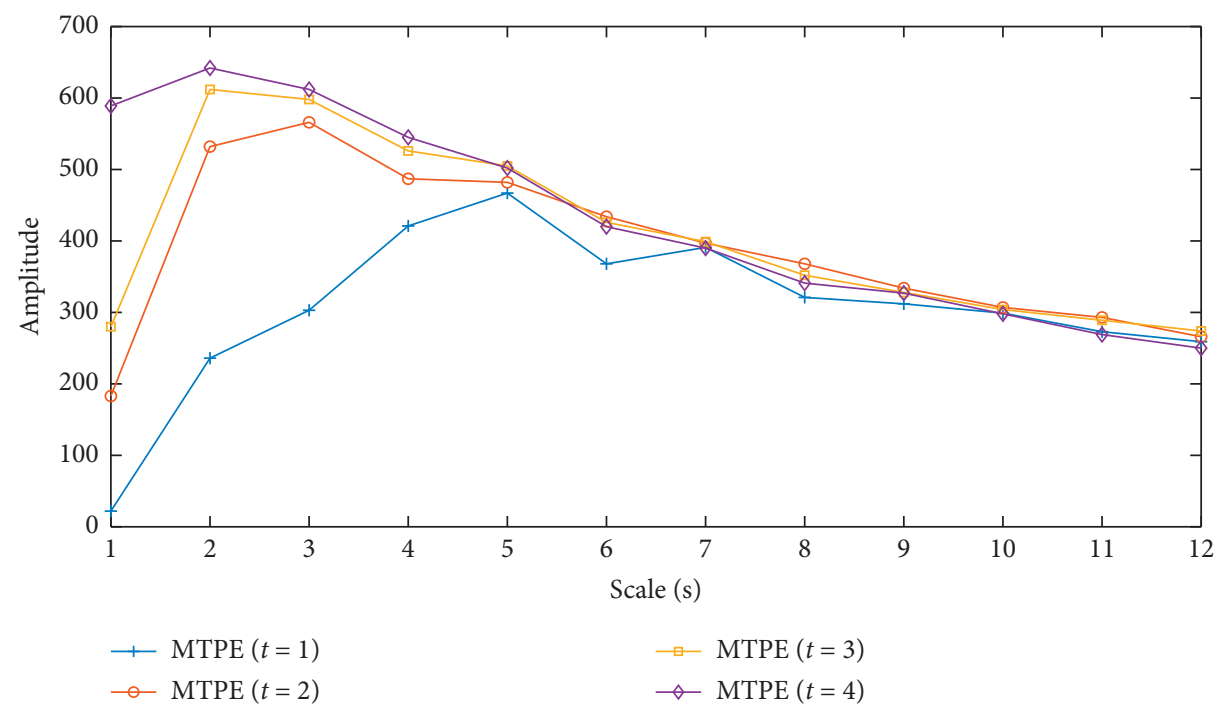

Figure 5: Effects of time delay $\tau$ on MTPEs.

Health stage division
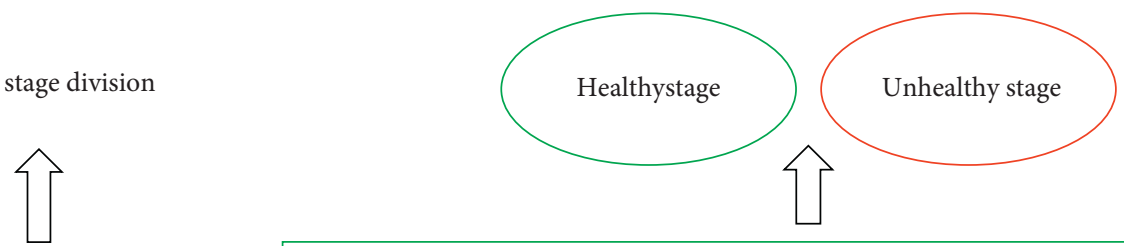

Healthy indicators
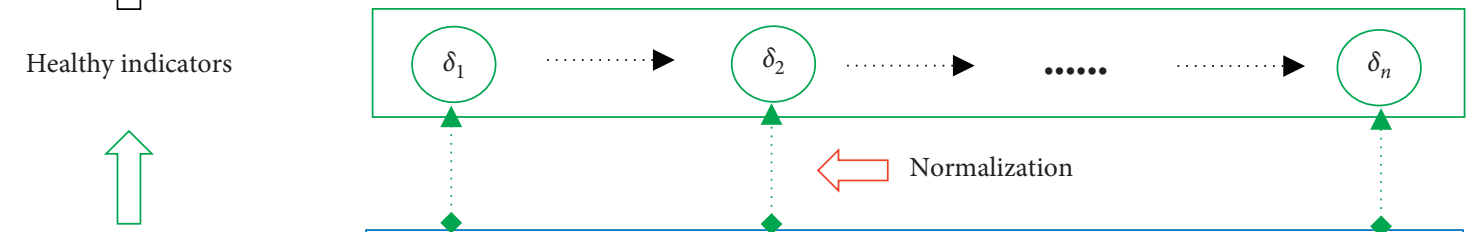

MTPES

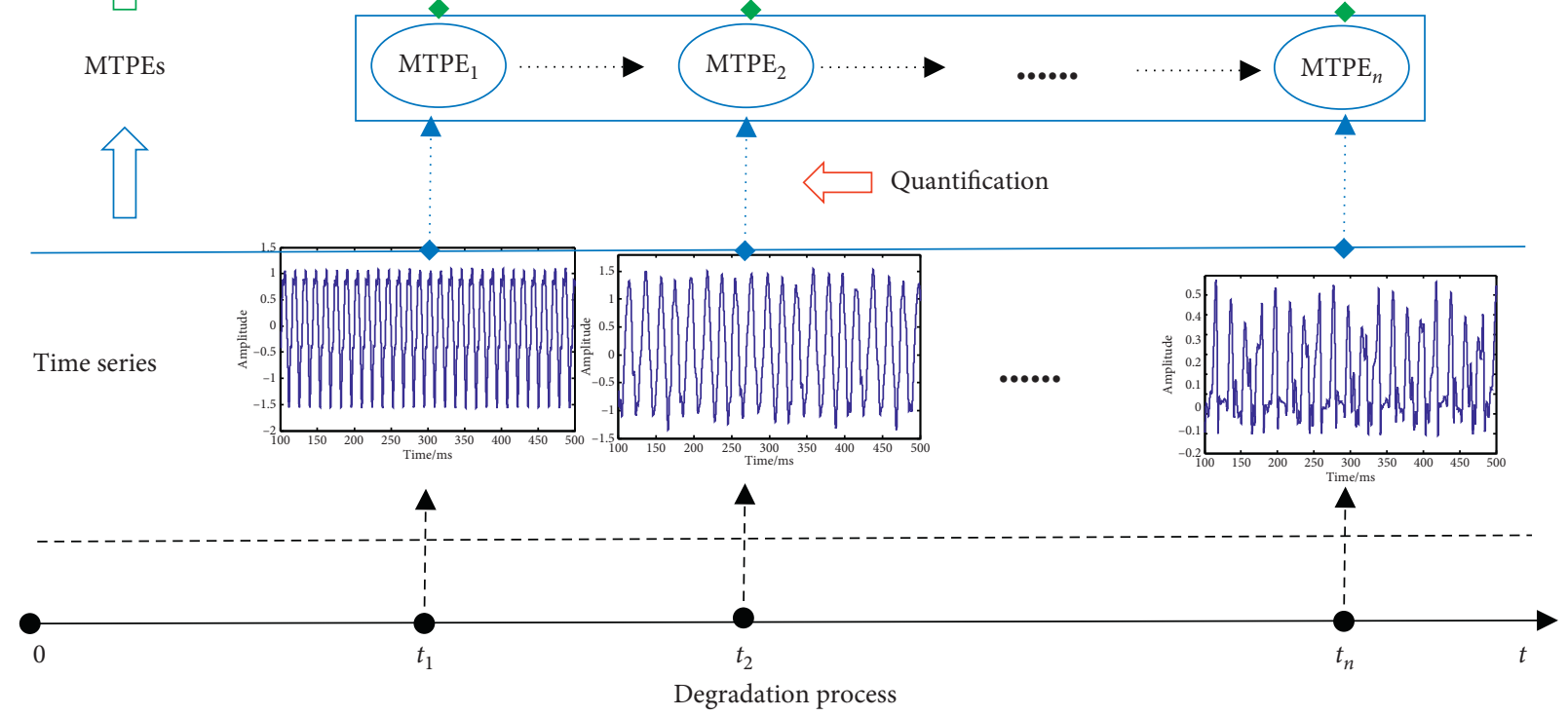

Figure 6: Procedures for performance degradation assessment.

signals at varying resolutions by decomposing the signals into their frequency bands. In this study, the Symmlet family of wavelet was chosen as the mother wavelet to implement the denoising. During the process of wavelet decomposition, the soft threshold was used and a denoised version of the signal was obtained using a wavelet coefficients threshold with a global positive threshold. The effects of denoising by Symmlet 4 wavelet and $\mathrm{db} 1$ wavelet with level 5 for a set of vibration signals are compared in Figure 8.

To verify the degradation trend of rolling bearing, several collected vibration signals (Figure 9) at different sampling times were transformed to frequency domains 


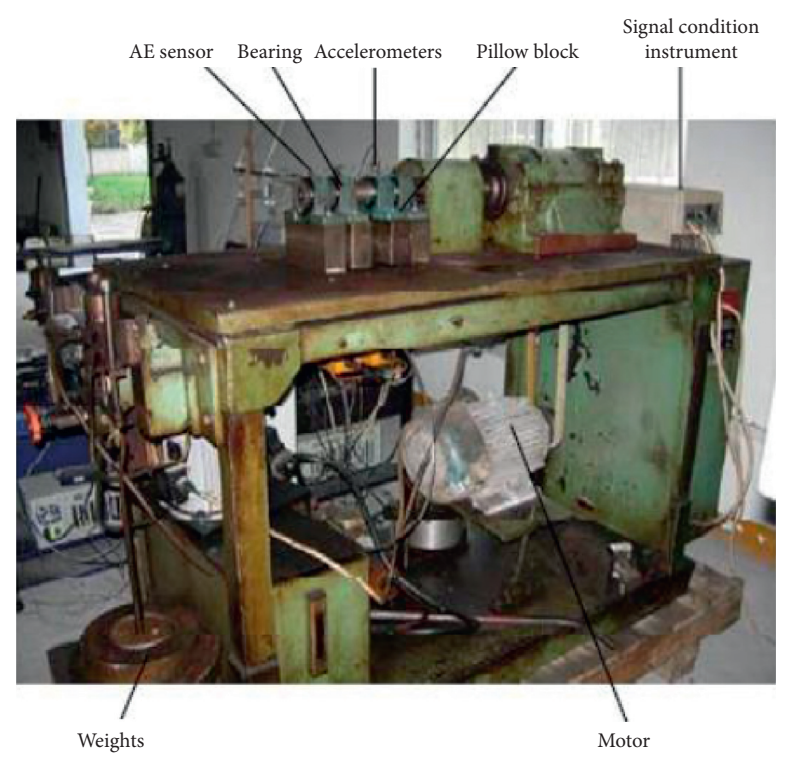

FIgURE 7: The ABLT-7 bearing test system used for the run to failure experiment.

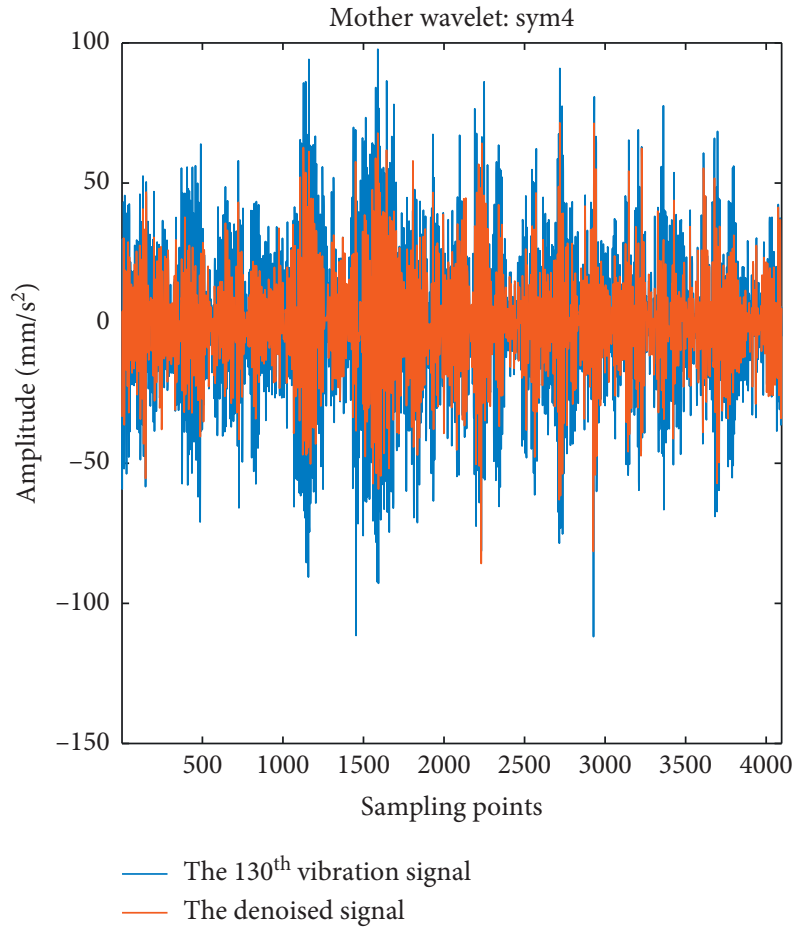

(a)

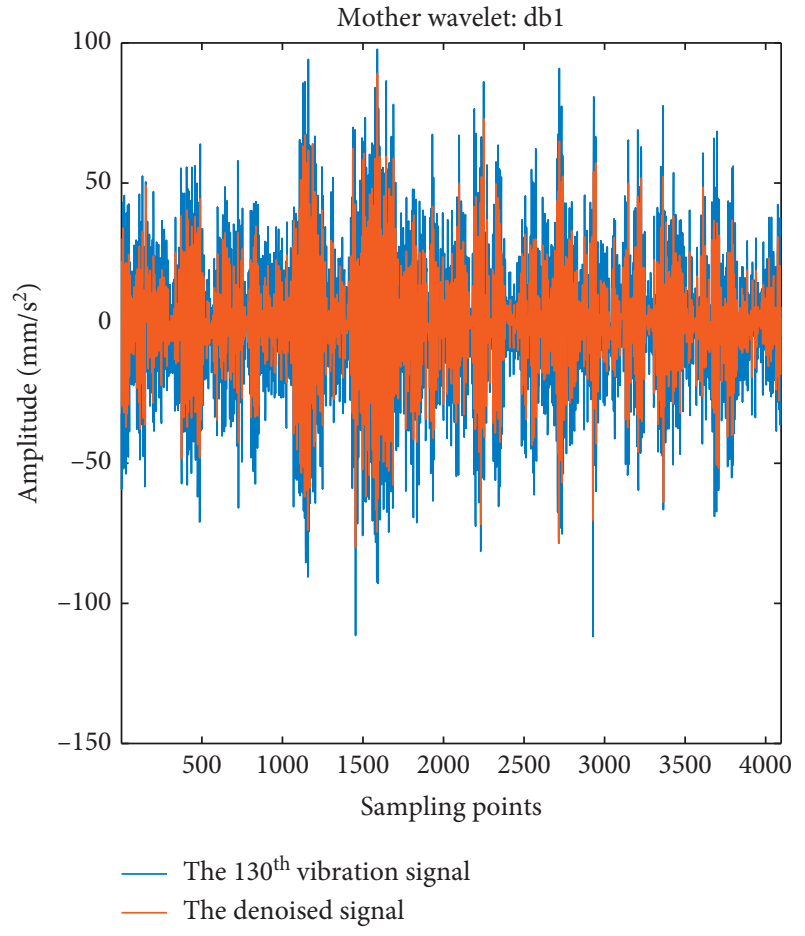

(b)

Figure 8: Comparisons of the wavelet denoising based on sym 4 and $\mathrm{db} 1$ mother wavelet.

using Fast Fourier Transform (FFT). The frequency spectrums of the vibration signals $\mathbf{X}_{20}, \mathbf{X}_{200}, \mathbf{X}_{380}, \mathbf{X}_{530}$ are shown in Figure 10. During the degradation process of the rolling bearing, more frequency components were aroused and the amplitudes of these frequencies were notably increased. Moreover, the dynamics of the rolling bearing during the degradation process became more complicated and nonlinear. In particular, the last vibration signal $\mathbf{X}_{530}$ had a great deal of harmonics in its frequency spectrum.

3.3. Healthy Indicators Construction. In this section, the health indicators of the rolling bearing were constructed based on the MTPEs of the vibration signals obtained during the process of degradation. For the measured vibration 

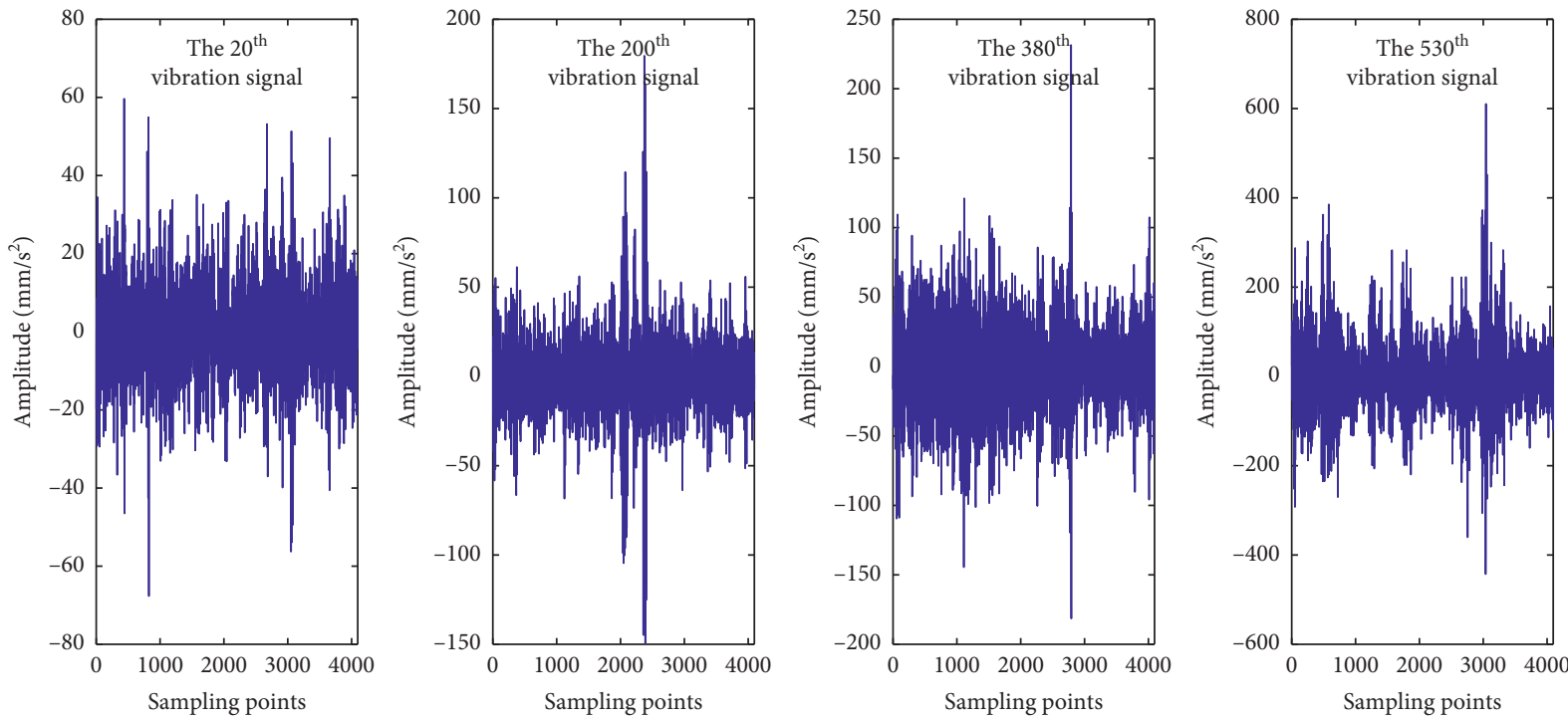

Figure 9: Vibration signals $\mathbf{X}_{20}, \mathbf{X}_{200}, \mathbf{X}_{380}, \mathbf{X}_{530}$.
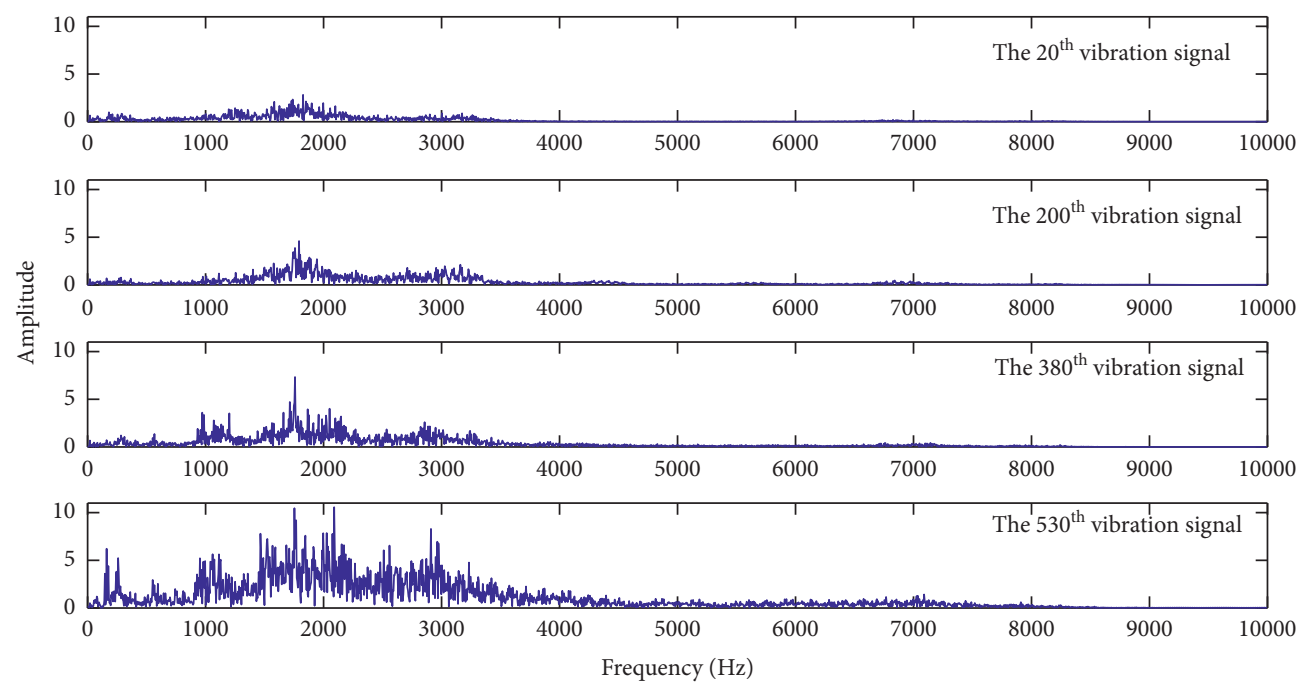

FIgURE 10: Frequency spectrum of vibration signals $\mathbf{X}_{20}, \mathbf{X}_{200}, \mathbf{X}_{380}, \mathbf{X}_{530}$.

signals $\mathbf{X}_{1}, \mathbf{X}_{2}, \ldots, \mathbf{X}_{530}$, a suitable entropy index $\mathbf{q}$ and scale s were determined to calculate the MTPEs.

To investigate the influence of these parameters, the relationship between health indicators and the entropy index $\mathbf{q}$ is given in Figure 11. The relationship between health indicators and scale $\mathbf{s}$ is shown in Figure 12. In the following discussion, entropy index $\mathbf{q}=-0.5$ and scale $\mathbf{s}=8$ were chosen to assess the status characterization for the rolling bearing during the degradation process. The embedding dimension $\mathbf{m}$ and time delay $\tau$ also had significant influences on the MTPE values during the degradation process of the rolling bearing. The results of the health indicators on these different parameters are shown in Figures 13 and 14, respectively.

Health indicators' trends were greatly changed with an embedding dimension of $\mathbf{m}=3$ (Figure 12) and a time delay of $\tau=1$ (Figure 14). Based on the above discussion, the embedding dimension and time delay were determined to be $\mathbf{m}=6$ and $\tau=3$. These results were similar to a previous analysis performed by permutation entropy [31]. The timedomain features (e.g., kurtosis, RMS, and skewness) were also computed and compared with the proposed health indicators in Figure 15. The kurtosis and skewness of the measured vibration signals fluctuated dramatically during the process of degradation for the rolling bearing; the RMS values were stable during this process.

However, the values of RMS decreased to a certain extent at the unhealthy stage of the rolling bearing. This gave an unreliable estimate of the health condition of the test bearing. The performance of prognostic HIs had a great influence on the complexity of prognostic modeling and the prediction accuracy. It should be noted that while the proposed health indicators seriously fluctuated at certain stages, the overall trend of the proposed HIs was easily divided into healthy and unhealthy stages. 


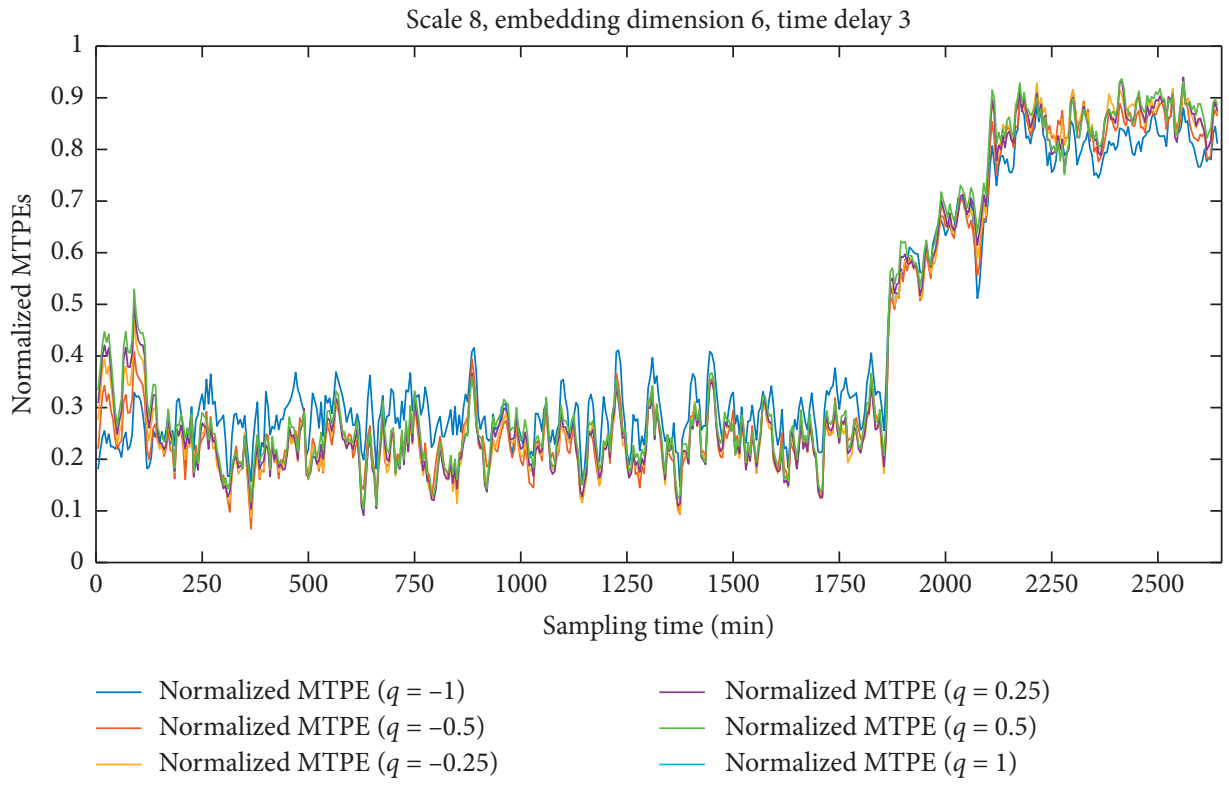

FIGURE 11: Results of health indicators on different entropy indices $\mathbf{q}$.

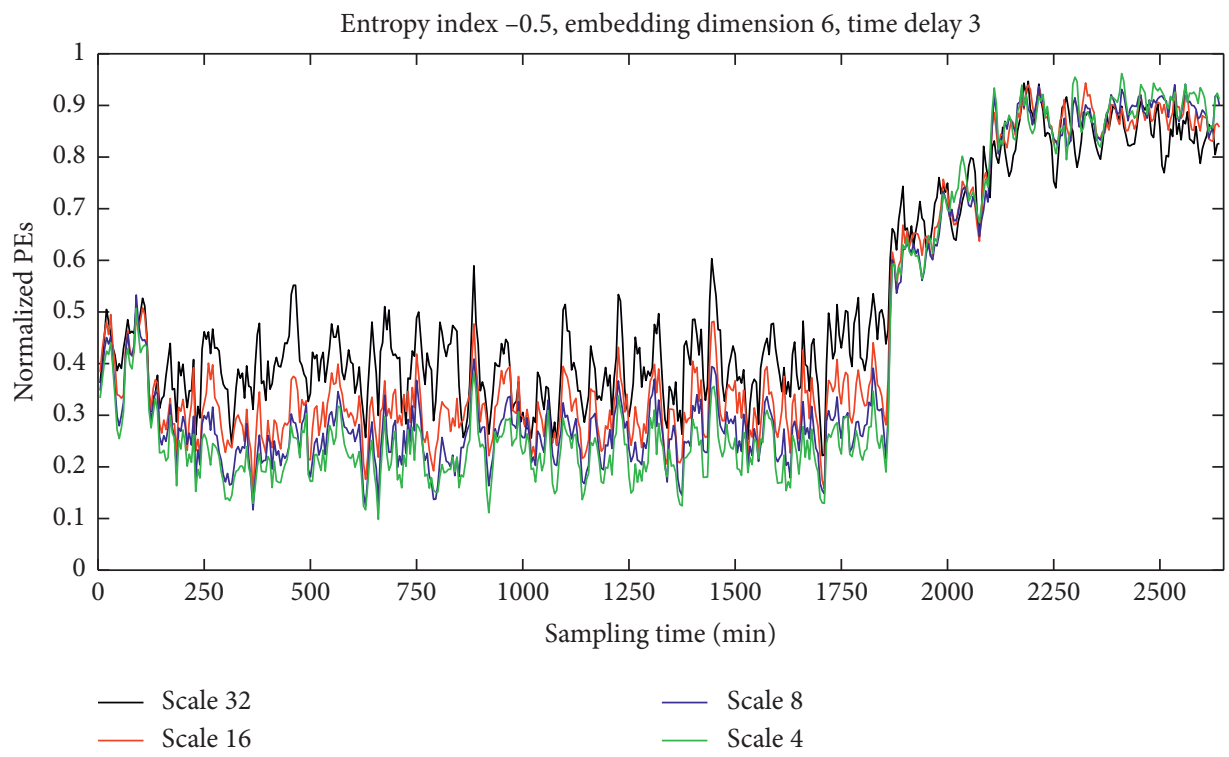

FIgURE 12: Results of health indicators on different scales $\mathbf{s}$.

3.4. Healthy Stage Division. With the varying degradation trends exhibited by the HIs, the degradation processes of the machinery should be divided into different HSs before RUL prediction. The aim of HS division is to divide the degradation processes of rolling processes into different HSs according to the varying trends of the HIs. Generally, the degradation trend of a rolling element bearing has two distinct stages-healthy and unhealthy $[1,8,38]$.

During the degradation process of the bearing, the proposed health indicators exhibited trends that were divided into two stages. A strategy for this two-stage division was used, whereby the HIs that exceeded a constant alarm threshold were identified [1]. Past work has used both constant [39-41] and adaptive [42,43] alarm thresholds to divide into healthy and unhealthy stages. This paper used the $3 \sigma$ interval as the alarm threshold to determine the initial starting point of the unhealthy stage; results are compared with the RMS values in Figure 16. Since no fault occurred in the rolling element bearing, the values of the proposed healthy indicators presented random fluctuation during the 


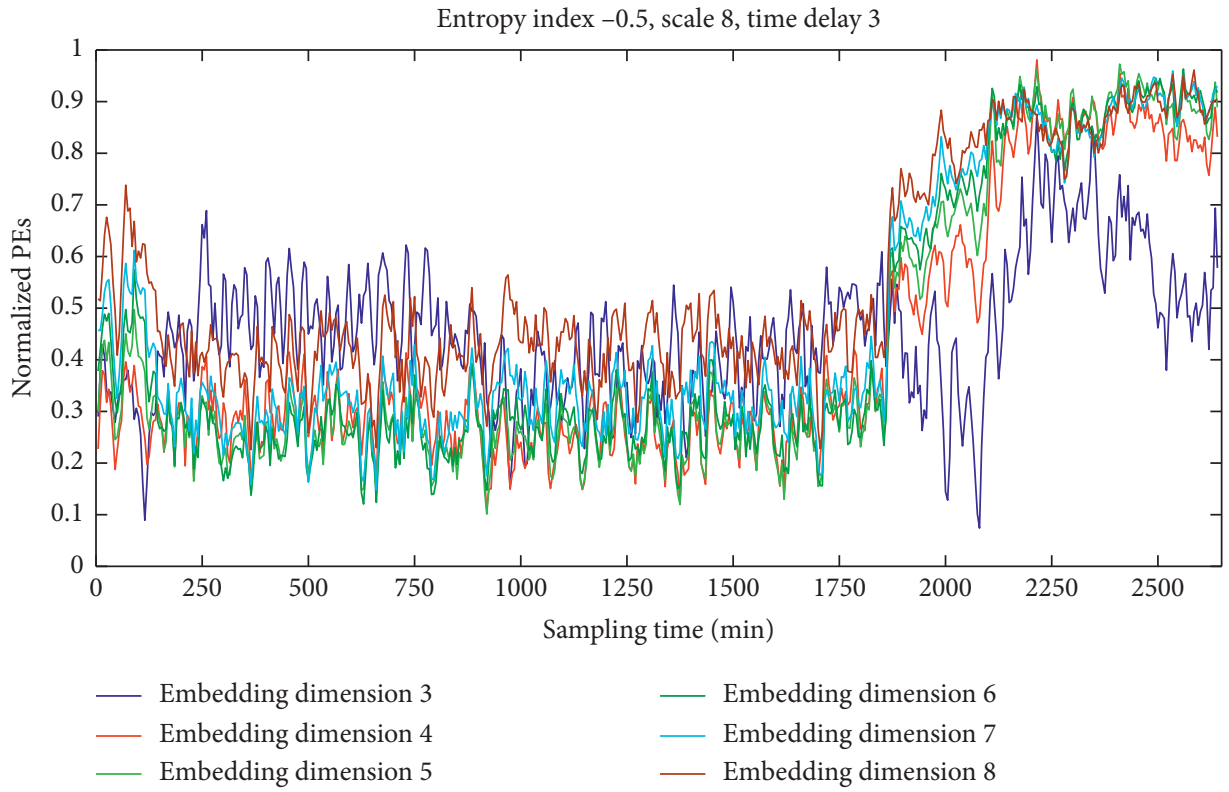

Figure 13: Results of health indicators on different embedding dimensions $\mathbf{m}$.

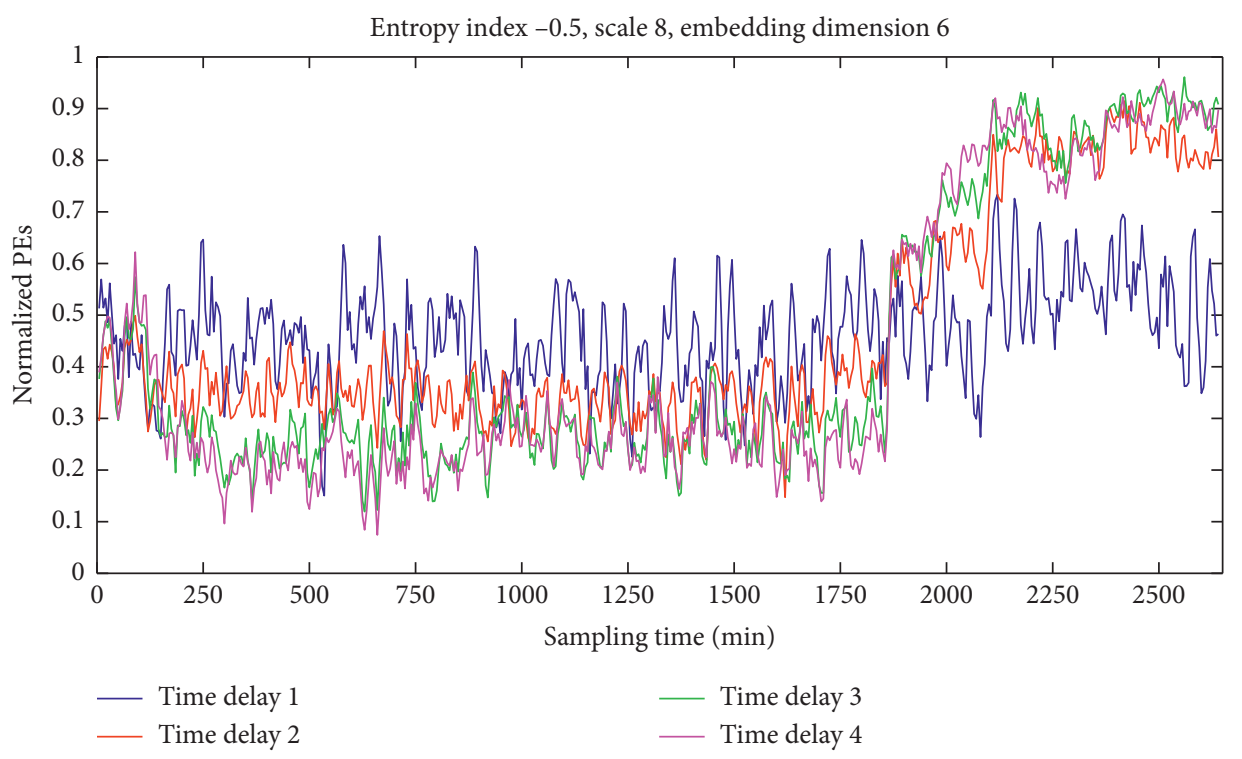

FIgURE 14: Results of health indicators on different time delays $\tau$.

healthy stage. Since there was no information regarding the degradation trend in the healthy stage, it was difficult and unnecessary to predict the RUL during this stage. However, HIs increased quickly with the deterioration of the tested rolling bearing at the unhealthy stage. However, the RMS values shown in Figure 16 indicate they were stable during the healthy stage. After that, they experienced an "increase- decrease-increase" trend during the unhealthy stage. Using the $3 \sigma$ interval as the alarm threshold to determine the initial point of the unhealthy stage, two HIs increased rapidly and reached the summit in a short time. However, the RMS values decreased during the last part of the unhealthy stage and this trend was difficult to model for RUI prediction. 


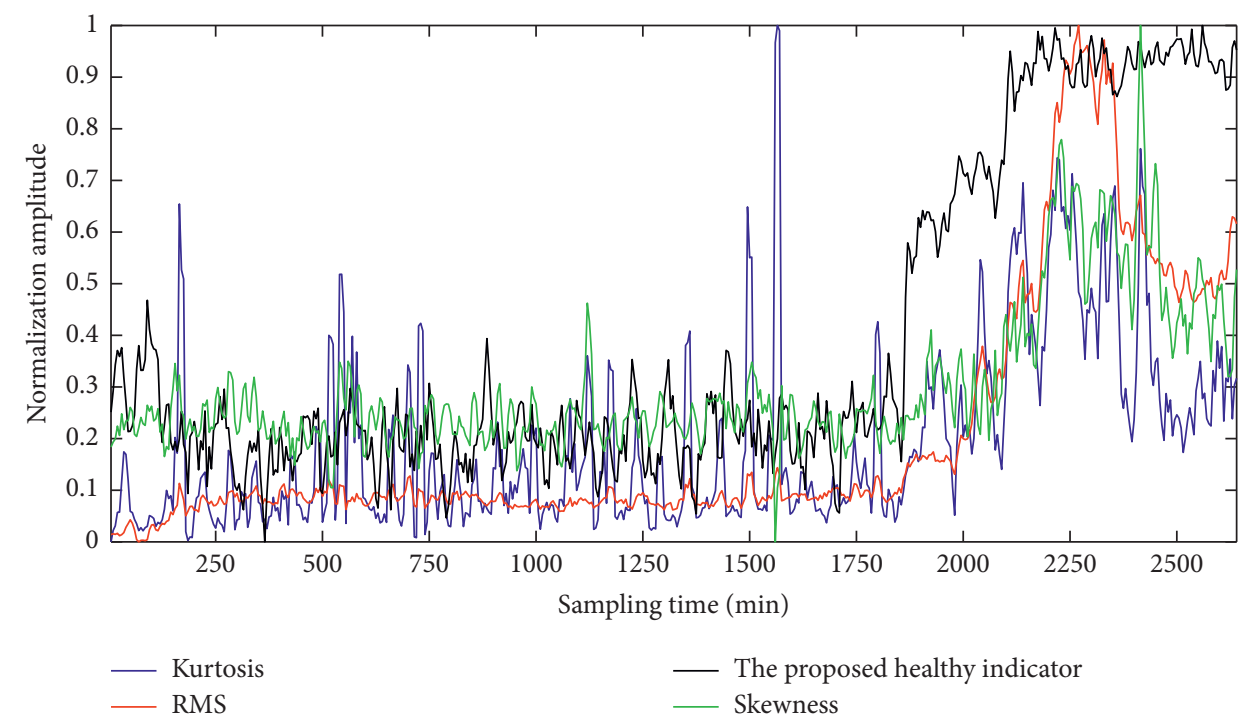

FIGURE 15: Comparison with three indicators: Kurtosis, skewness, and RMS.

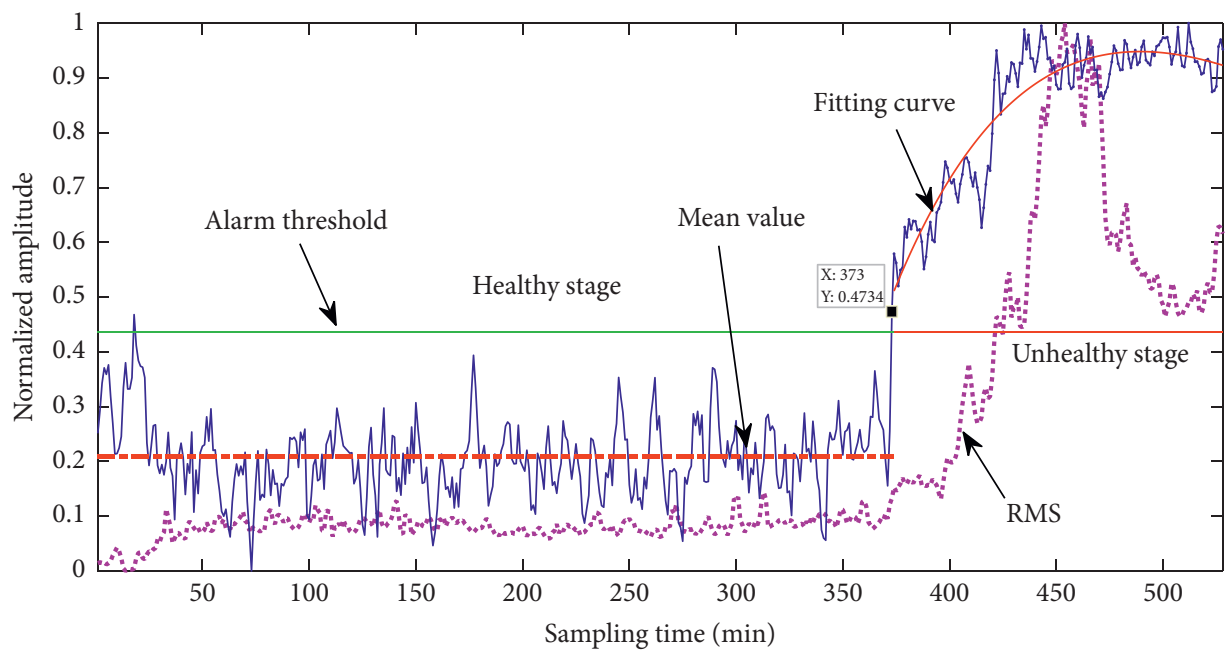

The proposed HIs

— Exponential function fitting

FIgURE 16: HS division for the proposed HIs based on the $3 \sigma$ alarm threshold.

\section{Conclusions}

This paper proposed the MTPE method for conditional monitoring during the degradation process of rotary machinery. The HIs were calculated by employing the MTPE to assess the complexity of the short and noising vibration signals. Using the $3 \sigma$ interval as the alarm threshold, two stages were identified-healthy and unhealthy. Since the values of MTPE were the function of the entropy index and scale, this method avoided the difficulties of constructing health indicators under changing working conditions. With the universality for assessing the complexity of permutated time series, this study provided a methodology using a novel complexity measure for conditional monitoring of rotary machinery.

\section{Data Availability}

The data and the MATLAB programs used to support the findings of this study are available from the corresponding author upon request.

\section{Conflicts of Interest}

The authors declare that they have no conflicts of interest.

\section{Acknowledgments}

This project was supported by the National Natural Science Foundation of China (Grant no. 51775182) and Guangdong 
Provincial Natural Science Foundation of China (2020B1515 120006).

\section{References}

[1] Y. Lei, N. Li, L. Guo, N. Li, T. Yan, and J. Lin, "Machinery health prognostics: a systematic review from data acquisition to RUL prediction," Mechanical Systems and Signal Processing, vol. 104, pp. 799-834, 2018.

[2] L. Cui, J. Huang, F. Zhang, and F. Chu, "HVSRMS localization formula and localization law: localization diagnosis of a ball bearing outer ring fault," Mechanical Systems and Signal Processing, vol. 120, no. 1, pp. 608-629, 2019.

[3] L. Cui, X. Wang, Y. Xu, H. Jiang, and J. Zhou, "A novel Switching Unscented Kalman Filter method for remaining useful life prediction of rolling bearing," Measurement, vol. 135, pp. 678-684, 2019.

[4] L. Cui, J. Huang, and F. Zhang, "Quantitative and localization diagnosis of a defective ball bearing based on vertical-horizontal synchronization signal analysis," IEEE Transactions on Industrial Electronics, vol. 64, no. 11, pp. 8695-8706, 2017.

[5] L. Cui, N. Wu, C. Ma, and H. Wang, "Quantitative fault analysis of roller bearings based on a novel matching pursuit method with a new step-impulse dictionary," Mechanical Systems and Signal Processing, vol. 68-69, pp. 34-43, 2016.

[6] M. Jiang, J. Wu, X. Peng, and X. Li, "Nonlinearity measure based assessment method for pedestal looseness of bearingrotor systems," Journal of Sound and Vibration, vol. 411, pp. 232-246, 2017.

[7] H. Qiu, J. Lee, J. Lin, and G. Yu, "Robust performance degradation assessment methods for enhanced rolling element bearing prognostics," Advanced Engineering Informatics, vol. 17, no. 3-4, pp. 127-140, 2003.

[8] L. Guo, N. Li, F. Jia, Y. Lei, and J. Lin, "A recurrent neural network based health indicator for remaining useful life prediction of bearings," Neurocomputing, vol. 240, pp. 98-109, 2017.

[9] B. Zhang, S. Zhang, and W. Li, "Bearing performance degradation assessment using long short-term memory recurrent network," Computers in Industry, vol. 106, pp. 14-29, 2019.

[10] Y. Liao, L. Zhang, and W. Li, "Regrouping particle swarm optimization based variable neural network for gearbox fault diagnosis," Journal of Intelligent \& Fuzzy Systems, vol. 34, no. 6, pp. 3671-3680, 2018.

[11] L. Liao, "Discovering prognostic features using genetic programming in remaining useful life prediction," IEEE Trans. Ind. Electron.vol. 61, no. 5, pp. 2464-2472, 2013.

[12] Z.-X. Zhang, X.-S. Si, and C.-H. Hu, "An age- and statedependent nonlinear prognostic model for degrading systems," IEEE Transactions on Reliability, vol. 64, no. 4, pp. 1214-1228, 2015.

[13] A. Malhi, R. Yan, and R. X. Gao, "Prognosis of defect propagation based on recurrent neural networks," IEEE Transactions on Instrumentation and Measurement, vol. 60, no. 3, pp. 703-711, 2011.

[14] H. Liao and Z. Tian, "A framework for predicting the remaining useful life of a single unit under time-varying operating conditions," IIE Transactions, vol. 45, no. 9, pp. 964-980, 2013.

[15] Z. Huang, Z. Xu, X. Ke, W. Wang, and Y. Sun, "Remaining useful life prediction for an adaptive skew-Wiener process model," Mechanical Systems and Signal Processing, vol. 87, pp. 294-306, 2017.
[16] Z. Huang, Z. Xu, W. Wang, and Y. Sun, "Remaining useful life prediction for a nonlinear heterogeneous wiener process model with an adaptive drift," IEEE Transactions on Reliability, vol. 64, no. 2, pp. 687-700, 2015.

[17] Z. Chen and W. Li, "Multisensor feature fusion for bearing fault diagnosis using sparse autoencoder and deep belief network," IEEE Transactions on Instrumentation and Measurement, vol. 66, no. 7, pp. 1693-1702, 2017.

[18] Y. Qian, R. Yan, and S. Hu, "Bearing degradation evaluation using recurrence quantification analysis and Kalman filter," IEEE Transactions on Instrumentation and Measurement, vol. 63, no. 11, pp. 2599-2610, 2014.

[19] T. Benkedjouh, K. Medjaher, N. Zerhouni, and S. Rechak, "Remaining useful life estimation based on nonlinear feature reduction and support vector regression," Engineering Applications of Artificial Intelligence, vol. 26, no. 7, pp. 17511760, 2013.

[20] H. Ocak, K. A. Loparo, and F. M. Discenzo, "Online tracking of bearing wear using wavelet packet decomposition and probabilistic modeling: a method for bearing prognostics," Journal of Sound and Vibration, vol. 302, no. 4-5, pp. 951-961, 2007.

[21] B. Keshtegar, M. Bagheri, C. W. Fei et al., "Multi-extremummodified response basis model for nonlinear response prediction of dynamic turbine blisk, Engineering with Computers," 2020.

[22] C.-W. Fei, H. Li, H.-T. Liu et al., "Enhanced network learning model with intelligent operator for the motion reliability evaluation of flexible mechanism," Aerospace Science and Technology, vol. 107, 2020.

[23] L. Han, C. Chen, T. Guo et al., "Probability-based service safety life prediction approach of raw and treated turbine blades regarding combined cycle fatigue," Aerospace Science and Technology, vol. 110, 2021.

[24] F. Jia, Y. Lei, J. Lin, X. Zhou, and N. Lu, "Deep neural networks: a promising tool for fault characteristic mining and intelligent diagnosis of rotating machinery with massive data," Mechanical Systems and Signal Processing, vol. 72-73, pp. 303-315, 2016.

[25] H. Shao, H. Jiang, F. Wang, and Y. Wang, "Rolling bearing fault diagnosis using adaptive deep belief network with dualtree complex wavelet packet," ISA Transactions, vol. 69, pp. 187-201, 2017.

[26] M. S. Kan, A. C. C. Tan, and J. Mathew, "A review on prognostic techniques for non-stationary and non-linear rotating systems," Mechanical Systems and Signal Processing, vol. 62-63, pp. 1-20, 2015.

[27] H. Hong and M. Liang, "Fault severity assessment for rolling element bearings using the Lempel-Ziv complexity and continuous wavelet transform," Journal of Sound and Vibration, vol. 320, no. 1-2, pp. 452-468, 2009.

[28] L. Cui, X. Gong, J. Zhang, and H. Wang, "Double-dictionary matching pursuit for fault extent evaluation of rolling bearing based on the Lempel-Ziv complexity," Journal of Sound and Vibration, vol. 385, pp. 372-388, 2016.

[29] R. Yan and R. X. Gao, "Approximate Entropy as a diagnostic tool for machine health monitoring," Mechanical Systems and Signal Processing, vol. 21, no. 2, pp. 824-839, 2007.

[30] C. Bandt and B. Pompe, "Permutation entropy: a natural complexity measure for time series," Mechanical Systems and Signal Processing, vol. 88, pp. 174102-174111, 2002.

[31] R. Yan, Y. Liu, and R. X. Gao, "Permutation entropy: a nonlinear statistical measure for status characterization of 
rotary machines," Mechanical Systems and Signal Processing, vol. 29, pp. 474-484, 2012.

[32] L. Zunino and C. W. Kulp, "Detecting nonlinearity in short and noisy time series using the permutation entropy," Physics Letters A, vol. 381, no. 42, pp. 3627-3635, 2017.

[33] C.-W. Fei, Y.-S. Choy, G.-C. Bai, and W.-Z. Tang, "Multifeature entropy distance approach with vibration and acoustic emission signals for process feature recognition of rolling element bearing faults," Structural Health Monitoring, vol. 17, no. 2 , pp. $156-168,2018$.

[34] C.-W. Fei and G.-C. Bai, "Wavelet correlation feature scale entropy and fuzzy support vector machine approach for aeroengine whole-body vibration fault diagnosis," Shock and Vibration, vol. 20, no. 2, pp. 341-349, 2013.

[35] Y. Gao, F. Villecco, M. Li, and W. Song, "Multi-scale permutation entropy based on improved LMD and HMM for rolling bearing diagnosis," Entropy, vol. 19, no. 4, p. 176, 2017.

[36] C. Li and P. Shang, "Multiscale Tsallis permutation entropy analysis for complex physiological time series," Physica A: Statistical Mechanics and Its Applications, vol. 523, pp. 10-20, 2019.

[37] Y. Cao, W. Tung, J. B. Gao, V. A. Protopopescu, and L. M. Hively, "Detecting dynamical changes in time series using the permutation entropy," Mechanical Systems and Signal Processing, vol. 70, p. 46217, 2004.

[38] Y. Wang, Y. Peng, Y. Zi, X. Jin, and K.-L. Tsui, "A two-stage data-driven-based prognostic approach for bearing degradation problem," IEEE Transactions on Industrial Informatics, vol. 12, no. 3, pp. 924-932, 2016.

[39] B. Zhang, C. Sconyers, C. Byington, R. Patrick, M. E. Orchard, and G. Vachtsevanos, "A probabilistic fault detection approach: application to bearing fault detection," IEEE Transactions on Industrial Electronics, vol. 58, no. 5, 2011.

[40] A. Ajami and M. Daneshvar, "Data driven approach for fault detection and diagnosis of turbine in thermal power plant using Independent Component Analysis (ICA)," International Journal of Electrical Power \& Energy Systems, vol. 43, no. 1, pp. 728-735, 2012.

[41] R. Li and D. He, "Rotational machine health monitoring and fault detection using EMD-based acoustic emission feature quantification," IEEE Transactions on Instrumentation and Measurement, vol. 61, no. 4, pp. 990-1001, 2012.

[42] A. Alkaya and İ. Eker, "Variance sensitive adaptive thresholdbased PCA method for fault detection with experimental application," ISA Transactions, vol. 50, no. 2, pp. 287-302, 2011.

[43] L. Hu, N. Hu, X. Zhang, F. Gu, and M. Gao, "Novelty detection methods for online health monitoring and post data analysis of turbopumps," Journal of Mechanical Science and Technology, vol. 27, no. 7, pp. 1933-1942, 2013. 